\title{
Theoretical models of sheep BSE reveal possibilities
}

\author{
But we must remember that these theories are based on speculation, not on fact.
}

Sir - We do not yet know whether bovine spongiform encephalopathy (BSE) entered the UK sheep population at the height of the epidemic among cattle and, if it did, what the likely effects would be or whether the BSE prion could have persisted in sheep. The one study aimed at illuminating this question has collapsed in what might generously be called a failure of experimental protocol (see Nature 413, 760; 2001). But if BSE did get into the sheep flock, it seems reasonable to assume that cases of variant Creutzfeldt-Jakob disease (vCJD) could result among humans.

Two new theoretical papers, one published in Science and the other in Nature, describe a range of possibilities for what might have happened in the past and what might be going on today.

Kao et al. (Science 23 November 2001; $10.11261 /$ science 1067475) use such data as are available on the amount of meat and bone-meal sheep ate and the dose-response relationship. They estimate that about $80 \%$ of the UK flock is susceptible to BSE, with variations among genotypes and locations. They conclude that between 10 and 1,500 sheep may have become infected in 1990 and that with limited maternal transmission fewer than 20 clinical cases a year of BSE (hidden among 10,000 or so sheep with scrapie) are now occurring. They suggest that a much larger epidemic in sheep could develop in the coming decades if BSE transmits horizontally, but their paper does not investigate this possibility in detail.

Ferguson et al. (Nature 9 January 2002; 10.1038 /nature709), in contrast, focus on horizontal transmission and the development of the epidemic in the next 80 years. They estimate the $95 \%$ confidence interval for future vCJD mortality to be 50-50,000 deaths considering exposure to bovine BSE alone, with the upper bound increasing to 150,000 once exposure to the worst-case ovine BSE scenario examined is included. Yet this statement phrased in terms of confidence intervals may unintentionally convey to readers an unjustified degree of statistical precision - an issue discussed by Graham Medley (Science 294, 1663-1664; 2001). These estimates derive from a valuable but speculative exploration of nonlinear epidemiological models whose parameters are no more than illustrative guesses. Some simplifying assumptions in the model of Ferguson et al., for example, are that infected flocks become immune for 20 years and that genetic variation in resistance is among rather than within flocks (onethird are assumed susceptible). These assumptions could have substantial implications for projections of what could happen.

The crucial question for policy-makers is what should be done to manage a purely theoretical risk that BSE is present in sheep? The draft UK government contingency plan, should BSE be found in the present-day flock, can be seen at www.defra.gov.uk/corporate/consult/ sheepbse/index.htm. Throughout Europe, current practice is to remove from the food chain sheep tissue that would carry infectivity if it was present (such as the brain and spinal cord of older sheep). But studies of experimental infection of sheep with BSE show that the current strategy still allows tissues that could harbour BSE to enter the food chain. Ferguson et al. consider the likely effects of removing more tissues and of earlier-age cut-offs. Again, given the uncertainties and assumptions, the quantitative details cannot be taken literally, although the theoretical risk might be reduced substantially by allowing only lambs under six months old into the food chain and by removing more of the tissues that could harbour infectious material. The option of testing sheep for transmissible spongiform encephalopathies as they enter the abattoir and keeping infected animals out of the food chain is still some way off, as a cheap test of proven reliability does not yet exist.

In the light of such scientific uncertainty there is no easy formula for deciding on the right level of precautionary risk management. Studies such as those of Kao et al. and Ferguson et al., which make varied theoretical assumptions, are useful in an open debate about policy. But their tentative nature must be kept in mind and the many uncertainties acknowledged. John R. Krebs, Robert M. May, Michael P. H. Stumpf

Department of Zoology, South Parks Road, Oxford OX1 3PS, UK

\section{Dropped genetics paper lacked scientific merit}

Sir - Even though the controversial withdrawal of a paper on the genetic relatedness of Palestinians and Jews by the journal Human Immunology (see Nature 414,382 ; 2001) is a minor episode compared with the tragedies caused by ethnic/religious conflicts over past decades, the issues involved are worth revisiting.

The stated purpose of the paper by Antonio Arnaiz-Villena et al. was to "examine the genetic relationships between the Palestinians and their neighbours (particularly the Jews) in order to: (1) discover the Palestinian origins, and (2) explain the historic basis of the present ... conflict between Palestinians and other Muslim countries with Israelite Jews”.

They conclude: "Jews and Palestinians share a very similar HLA genetic pool that supports a common ancient Canaanite origin. Therefore, the origin of the longlasting Jewish-Palestinian hostility is the fight for land in ancient times."

It is difficult to believe that knowledge of genes may help to explain the present conflict. Although population genetics can address issues of relatedness of populations, mating patterns, migrations and so on, obviously it cannot provide evidence about reasons for conflicts between people.

Our primary concern, however, is that the authors might be perceived to have been discriminated against for political, as opposed to legitimate scientific, reasons.

Even a cursory look at the paper's diagrams and trees immediately indicates that the authors make some extraordinary claims. They used a single genetic marker, HLA DRB1, for their analysis to construct a genealogical tree and map of 28 populations from Europe, the Middle East, Africa and Japan. Using results from the analysis of a single marker, particularly one likely to have undergone selection, for the purpose of reconstructing genealogies is unreliable and unacceptable practice in population genetics.

The limitations are made evident by the authors' extraordinary observations that Greeks are very similar to Ethiopians and east Africans but very distant from other south Europeans; and that the Japanese are nearly identical to west and south Africans. It is surprising that the authors were not puzzled by these anomalous results, which contradict history, geography, anthropology and all prior population-genetic studies of these groups. Surely the ordinary process of refereeing would have saved the field from this dispute.

We believe that the paper should have been refused for publication on the simple grounds that it lacked scientific merit.

Neil Risch ${ }^{\star}$, Alberto Piazza $\dagger$, L. Luca Cavalli-Sforza ${ }^{\star}$

${ }^{\star}$ Department of Genetics, Stanford University School of Medicine, Stanford, California 94305, USA

†Department of Genetics, Biology and Biochemistry, University of Torino, Via Santena 19,

10126 Torino, Italy 